\title{
Positive periodic solutions for multiparameter nonlinear differential systems with delays
}

Ruipeng Chen ${ }^{1 *}$ (D) and Xiaoya Li ${ }^{1}$

"Correspondence:

ruipengchen@126.com

'Department of Mathematics,

North Minzu University, Yinchuan,

P.R. China

\section{Springer}

\begin{abstract}
We establish several criteria for the existence of positive periodic solutions of the multi-parameter differential systems

$$
\left\{\begin{array}{l}
u^{\prime}(t)+a_{1}(t) g_{1}(u(t)) u(t)=\lambda b_{1}(t) f\left(u\left(t-\tau_{1}(t)\right), v\left(t-\zeta_{1}(t)\right)\right), \\
v^{\prime}(t)+a_{2}(t) g_{2}(v(t)) v(t)=\mu b_{2}(t) g\left(u\left(t-\tau_{2}(t)\right), v\left(t-\zeta_{2}(t)\right)\right),
\end{array}\right.
$$

where the functions $g_{1}, g_{2}:[0, \infty) \rightarrow[0, \infty)$ are assumed to be unbounded. The analysis in the paper relies on the classical fixed point index theory. Our main findings improve and complement some existing results in the literature.
\end{abstract}

MSC: $34 \mathrm{~B} 15$

Keywords: Positive periodic solutions; Existence; Multiparameter systems; Fixed point

\section{Introduction}

Let $\omega>0$ be a constant. In this article we shall seek some criterion to guarantee that the multiparameter system

$$
\left\{\begin{array}{l}
u^{\prime}(t)=a_{1}(t) g_{1}(u(t)) u(t)-\lambda b_{1}(t) f\left(u\left(t-\tau_{1}(t)\right), v\left(t-\zeta_{1}(t)\right)\right), \\
v^{\prime}(t)=a_{2}(t) g_{2}(v(t)) v(t)-\mu b_{2}(t) g\left(u\left(t-\tau_{2}(t)\right), v\left(t-\zeta_{2}(t)\right)\right)
\end{array}\right.
$$

admits a positive $\omega$-periodic solution, where the functions $a_{i}, b_{i}, \tau_{i}, \zeta_{i} \in C(\mathbb{R}, \mathbb{R})$ are $\omega$ periodic, and $g_{i} \in C([0, \infty),[0, \infty))$ are unbounded, $i=1,2$. In addition, we assume that the nonlinear terms $f, g \in C([0, \infty) \times[0, \infty),[0, \infty))$ and $\lambda, \mu$ are positive parameters.

Here a positive periodic solution of (1.1) means a solution $(u, v) \in E:=X^{2}$ of (1.1) satisfying $u>0, v>0$ on $[0, \omega]$, where

$$
X=\{x \in C(\mathbb{R}, \mathbb{R}): x(t+\omega)=x(t)\}
$$

(c) The Author(s) 2020. This article is licensed under a Creative Commons Attribution 4.0 International License, which permits use, sharing, adaptation, distribution and reproduction in any medium or format, as long as you give appropriate credit to the original author(s) and the source, provide a link to the Creative Commons licence, and indicate if changes were made. The images or other third party material in this article are included in the article's Creative Commons licence, unless indicated otherwise in a credit line to the material. If material is not included in the article's Creative Commons licence and your intended use is not permitted by statutory regulation or exceeds the permitted use, you will need to obtain permission directly from the copyright holder. To view a copy of this licence, visit http://creativecommons.org/licenses/by/4.0/. 
is a Banach space, and the norm of $x \in X$ is

$$
\|x\|=\max _{t \in[0, \omega]}|x(t)| .
$$

Moreover, for $(x, y) \in E$, we denote $\|(x, y)\|=\|x\|+\|y\|$, and write $(x, y) \geq(0,0)$ if $(x, y) \in E$ fulfills $x(t) \geq 0, y(t) \geq 0, t \in[0, \omega]$.

Obviously, the first equation of (1.1) reduces in some special circumstances to

$$
u^{\prime}(t)=a(t) g(u(t)) u(t)-\lambda b(t) f(u(t-\tau(t)))
$$

and when $\lambda=0, g(u) \equiv 1$, Eq. (1.2) becomes $u^{\prime}(t)=a(t) u(t)$, which is famous in Malthusian population dynamics. In recent decades, (1.2) has also been extensively applied to describe various physiological processes emerging in practical applications, for instance, the production of blood cells, respiration, cardiac arrhythmias, etc. One may refer to [16] and references therein. Nevertheless, the research work in the above mentioned papers is mainly dependent on the condition that $g(u)$ is positive and bounded, that is, there are constants $L>l>0$ such that $0<l \leq g(u) \leq L, u \in[0, \infty)$. Jin and Wang [7] have recently studied the spectral problem

$$
u^{\prime}(t)=a(t) e^{u(t)} u(t)-\lambda b(t) f(u(t-\tau(t)))
$$

and they obtained some existence results on positive periodic solutions by means of the fixed point theory. It is worth noting the function $e^{u}$ is unbounded on $[0, \infty)$. Since then, Eq. (1.2) has been extensively investigated under the more general case that $g(u)$ is unbounded on $[0, \infty)$, by applying the lower and upper solutions method, fixed point theory, and so on. See, for example, [7-10].

Besides, researchers have focused on the differential systems associated to (1.2), namely,

$$
u_{i}^{\prime}(t)=a_{i}(t) g_{i}\left(u_{i}(t)\right) u_{i}(t)-\lambda b_{i}(t) f_{i}\left(u_{1}(t), u_{2}(t), \ldots, u_{n}(t)\right), \quad i=1,2, \ldots, n .
$$

One can see [11-14] for some related results. However, in [11-13], the authors have only dealt with the special case $g_{i}\left(u_{i}\right) \equiv 1, i=1,2, \ldots, n$. Indeed in that case, the Green's function corresponding to $u_{i}^{\prime}(t)=a_{i}(t) u_{i}(t)$ is simple, and some suitable cones could be easily constructed. Furthermore, system (1.3) investigated in above papers includes only one positive parameter $\lambda$. Hence, it will be interesting to study the multiparameter systems (1.1) with $g_{i}(i=1,2)$ being unbounded. On the other hand, what is worth mentioning is that Zhang et al. [14] considered system (1.1) for the special case $g_{i} \equiv 1, i=1,2$, where nonlinearities $f(u, v)$ and $g(u, v)$ were assumed to be nondecreasing, and only the case $f(0,0)>0$, $g(0,0)>0$ was treated. Therefore, we want to know whether or not (1.1) has a positive periodic solution under more relaxed assumption $f(0,0)=0, g(0,0)=0$. In view of above reasons, we shall concentrate on the existence of positive periodic solutions for system (1.1) in the current paper, to further improve and generalize tho results in the literature. For this purpose, we assume

(C1) $a_{i}, b_{i}, \tau_{i}, \zeta_{i} \in C(\mathbb{R},[0, \infty))$ are $\omega$-periodic with $\int_{0}^{\omega} a_{i}(t) d t>0, \int_{0}^{\omega} b_{i}(t) d t>0, i=1,2$.

(C2) There is $l_{i}>0$ such that $0<l_{i} \leq g_{i}(s)<\infty, s \in[0, \infty)$.

(C3) $f, g \in C([0, \infty) \times[0, \infty),[0, \infty))$ with $f(u, v)>0, g(u, v)>0$ for $(u, v) \neq(0,0)$. 
Remark 1.1 For other research work on periodic solutions of functional differential equations and systems, we refer the readers to [15-17] and references therein.

The remainder of the paper is arranged as follows. In Sect. 2, we introduce some preliminaries needed in our proof. Section 3 is devoted to stating and proving our main findings. Meanwhile, some related results and remarks will be given.

\section{Preliminaries}

Recall that $E=X^{2}$ is the Banach space defined as in Sect. 1. We first give the following lemma.

Lemma 2.1 Assume (C1)-(C3). If $(u, v) \in E$ is a solution of $(1.1)$, then

$$
\begin{aligned}
& u(t)=\lambda \int_{t}^{t+\omega} G_{1}(t, s) b_{1}(s) f\left(u\left(s-\tau_{1}(s)\right), v\left(s-\zeta_{1}(s)\right)\right) d s, \\
& v(t)=\mu \int_{t}^{t+\omega} G_{2}(t, s) b_{2}(s) g\left(u\left(s-\tau_{2}(s)\right), v\left(s-\zeta_{2}(s)\right)\right) d s,
\end{aligned}
$$

where

$$
G_{1}(t, s)=\frac{e^{-\int_{t}^{s} a_{1}(\theta) g_{1}(u(\theta)) d \theta}}{1-e^{-\int_{0}^{\omega} a_{1}(\theta) g_{1}(u(\theta)) d \theta}}, \quad G_{2}(t, s)=\frac{e^{-\int_{t}^{s} a_{2}(\theta) g_{2}(v(\theta)) d \theta}}{1-e^{-\int_{0}^{\omega} a_{2}(\theta) g_{2}(\nu(\theta)) d \theta}}, \quad t \leq s \leq t+\omega .
$$

Proof Multiplying the both sides of the first equation of (1.1) with $e^{-\int_{0}^{t} a_{1}(s) g_{1}(u(s)) d s}$, we can obtain

$$
\left(u(t) e^{-\int_{0}^{t} a_{1}(s) g_{1}(u(s)) d s}\right)^{\prime}=-\lambda b_{1}(t) f\left(u\left(t-\tau_{1}(t)\right), v\left(t-\zeta_{1}(t)\right)\right) \cdot e^{-\int_{0}^{t} a_{1}(s) g_{1}(u(s)) d s} .
$$

Integrating above equation from $t$ to $t+\omega$ and by elementary calculation, we can easily get

$$
u(t)=\lambda \int_{t}^{t+\omega} G_{1}(t, s) b_{1}(s) f\left(u\left(s-\tau_{1}(s)\right), v\left(s-\zeta_{1}(s)\right)\right) d s .
$$

Similar evaluation shows

$$
v(t)=\mu \int_{t}^{t+\omega} G_{2}(t, s) b_{2}(s) g\left(u\left(s-\tau_{2}(s)\right), v\left(s-\zeta_{2}(s)\right)\right) d s .
$$

Let $q>0$ be a fixed constant. Then we can establish a series of lemmas required in the subsequent discussion.

Lemma 2.2 Assume (C1)-(C3). Let $\sigma_{i}=e^{-\int_{0}^{\omega} a_{i}(\theta) d \theta}, i=1,2$. Then for any $(u, v) \in$ E satisfying $(u, v) \geq(0,0)$ and $\|(u, v)\| \leq q$,

$$
0<\frac{\sigma_{i}^{g_{i}^{*}(q)}}{1-\sigma_{i}^{g_{i}^{*}(q)}} \leq G_{i}(t, s) \leq \frac{1}{1-\sigma_{i}^{g_{i *}(q)}}, \quad i=1,2,
$$


where

$$
g_{i}^{*}(q)=\max _{0 \leq s \leq q} g_{i}(s), \quad g_{i *}(q)=\min _{0 \leq s \leq q} g_{i}(s), \quad i=1,2 .
$$

Proof Clearly, for $(u, v) \in E$ with $(u, v) \geq(0,0)$ and $\|(u, v)\| \leq q$, we have $0 \leq u \leq\|u\| \leq q$. Thus,

$$
g_{1 *}(q) \leq g_{1}(u) \leq g_{1}^{*}(q)
$$

and then simple estimation shows (2.1) holds for $i=1$. The case $i=2$ is similar.

Defining for $i=1,2$,

$$
m_{i}(q)=\frac{\sigma_{i}^{g_{i}^{*}(q)}}{1-\sigma_{i}^{g_{i}^{*}(q)}}, \quad M_{i}(q)=\frac{1}{1-\sigma_{i}^{g_{i *}(q)}}, \quad \eta_{i}(q)=\frac{m_{i}(q)}{M_{i}(q)} .
$$

Then it is not hard to verify $\eta_{i}(q) \in(0,1)$, and accordingly,

$$
\eta(q):=\min \left\{\eta_{1}(q), \eta_{2}(q)\right\} \in(0,1)
$$

Set

$$
\begin{aligned}
& P=\{(u, v) \in E: u(t) \geq 0, v(t) \geq 0, t \in[0, \omega]\}, \\
& K_{q}=\{(u, v) \in P: u(t)+v(t) \geq \eta(q)\|(u, v)\|, t \in[0, \omega]\},
\end{aligned}
$$

and for $r>0$,

$$
\Omega_{r}=\left\{(u, v) \in K_{q}:\|(u, v)\|<r\right\}, \partial \Omega_{r}=\left\{(u, v) \in K_{q}:\|(u, v)\|=r\right\} .
$$

Then $P$ and $K_{q}$ are cones in $E$.

Lemma 2.3 Assume (C1)-(C3). Let $0<r \leq q$. Then for any $(u, v) \in \bar{\Omega}_{r}$,

$$
\frac{\sigma_{i}^{g_{i}^{*}(q)}}{1-\sigma_{i}^{g_{i}^{*}(q)}} \leq \frac{\sigma_{i}^{g_{i}^{*}(r)}}{1-\sigma_{i}^{g_{i}^{*}(r)}} \leq G_{i}(t, s) \leq \frac{1}{1-\sigma_{i}^{g_{i *}(r)}} \leq \frac{1}{1-\sigma_{i}^{g_{i *}(q)}}, \quad i=1,2 .
$$

Proof Similar to the proof of Lemma 2.2, we obtain for $t \leq s \leq t+\omega$,

$$
\frac{\sigma_{i}^{g_{i}^{*}(r)}}{1-\sigma_{i}^{g_{i}^{*}(r)}} \leq G_{i}(t, s) \leq \frac{1}{1-\sigma_{i}^{g_{i *}(r)}}, \quad i=1,2
$$

Moreover, since $\varphi(t):=\frac{\sigma_{i}^{t}}{1-\sigma_{i}^{t}}$ and $\psi(t):=\frac{1}{1-\sigma_{i}^{t}}$ are strictly decreasing on $[0, \infty)$, one can easily see that (2.2) holds true.

Define, for given $(u, v) \in E$,

$$
T_{\lambda, \mu}(u, v)(t)=\left(A_{\lambda}(u, v)(t), B_{\mu}(u, v)(t)\right)
$$


where

$$
A_{\lambda}(u, v)(t)=\lambda \int_{t}^{t+\omega} G_{1}(t, s) b_{1}(s) f\left(u\left(s-\tau_{1}(s)\right), v\left(s-\zeta_{1}(s)\right)\right) d s
$$

and

$$
B_{\mu}(u, v)(t)=\mu \int_{t}^{t+\omega} G_{2}(t, s) b_{2}(s) g\left(u\left(s-\tau_{2}(s)\right), v\left(s-\zeta_{2}(s)\right)\right) d s .
$$

Then we have

Lemma 2.4 Assume (C1)-(C3) and $0<r \leq q$. Then $T_{\lambda, \mu}\left(\bar{\Omega}_{r}\right) \subseteq K_{q}$ and $T_{\lambda, \mu}: \bar{\Omega}_{r} \rightarrow K_{q}$ is completely continuous.

Proof For $(u, v) \in \bar{\Omega}_{r}$, we can deduce from Lemma 2.3 that

$$
\begin{aligned}
A_{\lambda}(u, v)(t) & =\lambda \int_{t}^{t+\omega} G_{1}(t, s) b_{1}(s) f\left(u\left(s-\tau_{1}(s)\right), v\left(s-\zeta_{1}(s)\right)\right) d s \\
& \leq \lambda \frac{1}{1-\sigma_{1}^{g_{1 *}(r)}} \int_{0}^{\omega} b_{1}(s) f\left(u\left(s-\tau_{1}(s)\right), v\left(s-\zeta_{1}(s)\right)\right) d s,
\end{aligned}
$$

which yields

$$
\left\|A_{\lambda}(u, v)\right\| \leq \lambda \frac{1}{1-\sigma_{1}^{g_{1 *}(r)}} \int_{0}^{\omega} b_{1}(s) f\left(u\left(s-\tau_{1}(s)\right), v\left(s-\zeta_{1}(s)\right)\right) d s .
$$

Meanwhile, (2.2) implies

$$
\begin{aligned}
A_{\lambda}(u, v)(t) & \geq \lambda \frac{\sigma_{1}^{g_{1}^{*}(r)}}{1-\sigma_{1}^{g_{1}^{*}(r)}} \int_{0}^{\omega} b_{1}(s) f\left(u\left(s-\tau_{1}(s)\right), v\left(s-\zeta_{1}(s)\right)\right) d s \\
& =\lambda \frac{\sigma_{1}^{g_{1}^{*}(r)}\left(1-\sigma_{1}^{g_{1 *}(r)}\right)}{1-\sigma_{1}^{g_{1}^{*}(r)}} \cdot \frac{1}{1-\sigma_{1}^{g_{1 *}(r)}} \int_{0}^{\omega} b_{1}(s) f\left(u\left(s-\tau_{1}(s)\right), v\left(s-\zeta_{1}(s)\right)\right) d s \\
& \geq \frac{\sigma_{1}^{g_{1}^{*}(r)}\left(1-\sigma_{1}^{g_{1 *}(r)}\right)}{1-\sigma_{1}^{g_{1}^{*}(r)}}\|A(u, v)\| \\
& \geq \eta_{1}(q)\left\|A_{\lambda}(u, v)\right\| \\
& \geq \eta(q)\left\|A_{\lambda}(u, v)\right\| .
\end{aligned}
$$

In an analogous manner, we get

$$
B_{\mu}(u, v)(t) \geq \eta(q)\left\|B_{\mu}(u, v)\right\|, \quad(u, v) \in \bar{\Omega}_{r} .
$$

Hence $T_{\lambda, \mu}\left(\bar{\Omega}_{r}\right) \subseteq K_{q}$. The completely continuity of $T_{\lambda, \mu}$ is obvious.

It is obvious that if $(u, v)$ is a fixed point of the completely continuous operator $T_{\lambda, \mu}$ in $K_{q}$, then $(u, v)$ is a positive periodic solution of (1.1). We conclude this section by giving the main tool employed in proving our main results. 
Lemma 2.5 ([18, 19]) Assume $E$ is a Banach space and $K \subseteq E$ is a cone. For $r>0$, let $K_{r}=\{u \in K:\|u\|<r\}$ and $\partial K_{r}=\{u \in K:\|u\|=r\}$. Suppose $T: \bar{K}_{r} \rightarrow K$ is a completely continuous operator satisfying $T u \neq u, u \in \partial K_{r}$. Then

(i) If $\|T u\|<\|u\|, u \in \partial K_{r}$, then $i\left(T, \bar{K}_{r}, K\right)=1$;

(ii) If $\|T u\|>\|u\|, u \in \partial K_{r}$, then $i\left(T, \bar{K}_{r}, K\right)=0$.

\section{Main results}

Let

$$
f_{0}=\lim _{(u, v) \rightarrow 0} \frac{f(u, v)}{u+v}, \quad g_{0}=\lim _{(u, v) \rightarrow 0} \frac{g(u, v)}{u+v} .
$$

Theorem 3.1 Assume (C1)-(C3) hold and $f_{0}=0=g_{0}$. Then for every $q>0$, there is a constant $\gamma_{q}>0$ such that for all $\lambda, \mu>\gamma_{q}$, system (1.1) admits a positive periodic solution $(u, v)$ satisfying $\|(u, v)\| \leq q$.

Proof Choose $r_{1}=q$ and define

$$
\begin{aligned}
& \psi_{f}(q)=\min \{f(u, v): \eta(q) q \leq u+v \leq q\} \\
& \psi_{g}(q)=\min \{g(u, v): \eta(q) q \leq u+v \leq q\} .
\end{aligned}
$$

Take

$$
\gamma_{q}=q \cdot \max \left\{\frac{1}{2 \psi_{f}(q) m_{1}(q) \int_{0}^{\omega} b_{1}(s) d s}, \frac{1}{2 \psi_{g}(q) m_{2}(q) \int_{0}^{\omega} b_{2}(s) d s}\right\} .
$$

By Lemma 2.4, we know $T_{\lambda, \mu}\left(\bar{\Omega}_{q}\right) \subseteq K_{q}$ and $T_{\lambda, \mu}: \bar{\Omega}_{q} \rightarrow K_{q}$ is completely continuous. Fix $\lambda, \mu>\gamma_{q}$. Then for $(u, v) \in \partial \Omega_{q}$, we have $\eta(q) q \leq u+v \leq q$, and so

$$
\begin{aligned}
A_{\lambda}(u, v)(t) & \geq \lambda m_{1}(q) \int_{0}^{\omega} b_{1}(s) f\left(u\left(s-\tau_{1}(s)\right), v\left(s-\zeta_{1}(s)\right)\right) d s \\
& =\lambda m_{1}(q) \psi_{f}(q) \cdot \int_{0}^{\omega} b_{1}(s) d s \\
& >\frac{q}{2}=\frac{\|(u, v)\|}{2}
\end{aligned}
$$

which implies

$$
\left\|A_{\lambda}(u, v)\right\|>\frac{\|(u, v)\|}{2}, \quad(u, v) \in \partial \Omega_{q}
$$

Similarly,

$$
\left\|B_{\mu}(u, v)\right\|>\frac{\|(u, v)\|}{2}, \quad(u, v) \in \partial \Omega_{q} .
$$

Hence $\left\|T_{\lambda, \mu}(u, v)\right\|>\|(u, v)\|$ on $\partial \Omega_{q}$, and then Lemma 2.5 gives $i\left(T_{\lambda, \mu}, \bar{\Omega}_{q}, K_{q}\right)=0$.

On the other hand, since $f_{0}=g_{0}=0$, there exists a constant $r_{2}$ with $0<r_{2}<q$, such that for $(u, v)$ satisfying $0<u+v \leq r_{2}$,

$$
f(u, v) \leq \varepsilon(u+v), \quad g(u, v) \leq \varepsilon(u+v),
$$


where $\varepsilon>0$ is a constant satisfying

$$
\frac{2 \lambda \varepsilon \int_{0}^{\omega} b_{1}(s) d s}{1-\sigma_{1}^{g_{1}^{*}(q)}}<1, \quad \frac{2 \mu \varepsilon \int_{0}^{\omega} b_{2}(s) d s}{1-\sigma_{2}^{g_{2}^{*}(q)}}<1
$$

For $(u, v) \in \partial \Omega_{r_{2}}$, we can deduce by (2.2) and (3.1) that

$$
\begin{aligned}
A_{\lambda}(u, v)(t) & \leq \lambda \frac{1}{1-\sigma_{1}^{g_{1 *}\left(r_{2}\right)}} \int_{t}^{t+\omega} b_{1}(s) f\left(u\left(s-\tau_{1}(s)\right), v\left(s-\zeta_{1}(s)\right)\right) d s \\
& \leq \frac{\lambda \varepsilon}{1-\sigma_{1}^{g_{1 *}(q)}} \cdot \int_{0}^{\omega} b_{1}(s) d s \cdot\|(u, v)\| \\
& <\frac{\|(u, v)\|}{2}
\end{aligned}
$$

and hence

$$
\left\|A_{\lambda}(u, v)\right\|<\frac{\|(u, v)\|}{2}, \quad(u, v) \in \partial \Omega_{r_{2}} .
$$

In an analogous way, we get

$$
\left\|B_{\mu}(u, v)\right\|<\frac{\|(u, v)\|}{2}, \quad(u, v) \in \partial \Omega_{r_{2}} .
$$

Thus $\left\|T_{\lambda, \mu}(u, v)\right\|<\|(u, v)\|$ on $\partial \Omega_{r_{2}}$. Lemma 2.5 ensures $i\left(T_{\lambda, \mu}, \bar{\Omega}_{r_{2}}, K_{q}\right)=1$.

Consequently, $i\left(T_{\lambda, \mu}, \bar{\Omega}_{q} \backslash \Omega_{r_{2}}, K_{q}\right)=-1$. Therefore, $T_{\lambda, \mu}$ possesses a fixed point $(u, v)$ in $\bar{\Omega}_{q} \backslash \Omega_{r_{2}}$, and system (1.1) has a positive periodic solution $(u, v)$ with $\|(u, v)\| \leq q$.

Theorem 3.2 Assume (C1)-(C3) hold and $f_{0}=\infty$. Then for every $q>0$, there is a constant $\gamma_{q}>0$ such that for all $\lambda, \mu<\gamma_{q}$, system (1.1) admits a positive periodic solution $(u, v)$ satisfying $\|(u, v)\| \leq q$.

Proof Fix $r_{1}=q$ and set

$$
\begin{aligned}
& \Psi_{f}(q)=\max \{f(u, v): \eta(q) q \leq u+v \leq q\} \\
& \Psi_{g}(q)=\max \{g(u, v): \eta(q) q \leq u+v \leq q\} .
\end{aligned}
$$

Define

$$
\gamma_{q}=q \cdot \min \left\{\frac{1}{2 \Psi_{f}(q) M_{1}(q) \int_{0}^{\omega} b_{1}(s) d s}, \frac{1}{2 \Psi_{g}(q) M_{2}(q) \int_{0}^{\omega} b_{2}(s) d s}\right\} .
$$

By Lemma 2.4, $T_{\lambda, \mu}\left(\bar{\Omega}_{q}\right) \subseteq K_{q}$ and $T_{\lambda, \mu}: \bar{\Omega}_{q} \rightarrow K_{q}$ is completely continuous. Thus, for fixed $\lambda, \mu<\gamma_{q}$ and $(u, v) \in \partial \Omega_{q}$,

$$
\begin{aligned}
A_{\lambda}(u, v)(t) & \leq \lambda M_{1}(q) \int_{0}^{\omega} b_{1}(s) f\left(u\left(s-\tau_{1}(s)\right), v\left(s-\zeta_{1}(s)\right)\right) d s \\
& =\lambda M_{1}(q) \psi_{f}(q) \cdot \int_{0}^{\omega} b_{1}(s) d s \\
& <\frac{q}{2}=\frac{\|(u, v)\|}{2},
\end{aligned}
$$


and then

$$
\left\|A_{\lambda}(u, v)\right\|<\frac{\|(u, v)\|}{2}, \quad(u, v) \in \partial \Omega_{q} .
$$

By a similar argument, we can also obtain

$$
\left\|B_{\mu}(u, v)\right\|<\frac{\|(u, v)\|}{2}, \quad(u, v) \in \partial \Omega_{q} .
$$

Therefore, $\left\|T_{\lambda, \mu}(u, v)\right\|<\|(u, v)\|$ for $(u, v) \in \partial \Omega_{q}$. Using Lemma 2.5 again, we can easily get $i\left(T_{\lambda, \mu}, \bar{\Omega}_{q}, K_{q}\right)=1$.

By the assumption $f_{0}=\infty$, there exists a constant $r_{2} \in(0, q)$, such that for $(u, v)$ satisfying $0<u+v \leq r_{2}$,

$$
f(u, v) \geq \Upsilon(u+v)
$$

where $\Upsilon>0$ satisfies

$$
\lambda \Upsilon \eta(q) \frac{\sigma_{1}^{g_{1}^{*}(q)}}{1-\sigma_{1}^{g_{1}^{*}(q)}} \int_{0}^{\omega} b_{1}(s) d s>1 .
$$

Thus for $(u, v) \in \partial \Omega_{r_{2}}$, we get by (2.2) and (3.2) that

$$
\begin{aligned}
A_{\lambda}(u, v)(t) & \geq \lambda \frac{\sigma_{1}^{g_{1}^{*}\left(r_{2}\right)}}{1-\sigma_{1}^{g_{1}^{*}\left(r_{2}\right)}} \int_{0}^{\omega} b_{1}(s) f\left(u\left(s-\tau_{1}(s)\right), v\left(s-\zeta_{1}(s)\right)\right) d s \\
& \geq \lambda \Upsilon r \eta(q) \frac{\sigma_{1}^{g_{1}^{*}(q)}}{1-\sigma_{1}^{g_{1}^{*}(q)}} \int_{0}^{\omega} b_{1}(s) d s \cdot\|(u, v)\| \\
& >\|(u, v)\|,
\end{aligned}
$$

which means $\left\|A_{\lambda}(u, v)\right\|>\|(u, v)\|$ on $\partial \Omega_{r_{2}}$. Hence

$$
\left\|T_{\lambda, \mu}(u, v)\right\| \geq\left\|A_{\lambda}(u, v)\right\|>\|(u, v)\|, \quad(u, v) \in \partial \Omega_{r_{2}}
$$

and Lemma 2.5 again implies $i\left(T_{\lambda, \mu}, \bar{\Omega}_{r_{2}}, K_{q}\right)=0$.

Consequently, $i\left(T_{\lambda, \mu}, \bar{\Omega}_{q} \backslash \Omega_{r_{2}}, K_{q}\right)=1$. Thus, $T_{\lambda, \mu}$ has a fixed point $(u, v)$ in $\bar{\Omega}_{q} \backslash \Omega_{r_{2}}$, and (1.1) has a positive periodic solution $(u, v)$ with $\|(u, v)\| \leq q$.

Similarly to Theorems 3.1 and 3.2, we can prove the following

Theorem 3.3 Assume (C1)-(C3) and $g_{0}=\infty$. Then for every $q>0$, there is a constant $\gamma_{q}>$ 0 such that for all $\lambda, \mu<\gamma_{q}$, system (1.1) admits a positive periodic solution $(u, v)$ satisfying $\|(u, v)\| \leq q$.

Remark 3.1 Clearly, the results of Theorems 3.1-3.3 generalize and complement the corresponding ones in $[7,9,12-14]$. 
To illustrate our main findings, we may choose $\omega=2 \pi$ and $\tau_{i} \equiv 0, \zeta_{i} \equiv 0(i=1,2)$ in the subsequent discussion. Let

$$
\begin{array}{ll}
a_{1}(t)=\sin t+1, & a_{2}(t)=\sin t+2, \quad t \in[0,2 \pi], \\
b_{1}(t)=\cos t+2, & b_{2}(t)=\cos t+1, \quad t \in[0,2 \pi] .
\end{array}
$$

Then it is not hard to check that $(\mathrm{C} 1)$ is satisfied. Moreover, define

$$
g_{1}(s)=e^{s}, \quad g_{2}(s)=2 e^{s}, \quad s \in[0, \infty)
$$

then there are constants $l_{1}=1$ and $l_{2}=2$ such that

$$
0<1=l_{1} \leq g_{1}(s)<\infty, \quad 0<2=l_{2} \leq g_{2}(s)<\infty, \quad s \in[0, \infty) .
$$

Hence (C2) is also satisfied.

Example 3.1 For $(u, v) \in[0, \infty) \times[0, \infty)$, let

$$
f(u, v)=3(u+v)^{2}\left(u^{2}+v^{2}+1\right)^{2}, \quad g(u, v)=2(u+v)^{4}\left(u^{2}+v^{2}+5\right)^{2} .
$$

Then $f, g \in C([0, \infty) \times[0, \infty),[0, \infty))$ with $f(u, v)>0, g(u, v)>0$ for $(u, v) \neq(0,0)$. Thus (C3) holds true. Furthermore, simple calculation gives $f_{0}=0=g_{0}$. Consequently, the results of Theorem 3.1 are valid.

Example 3.2 We shall follow the same notations and definitions as before. Let us redefine

$$
f(u, v)=\sqrt{u+v} \cdot\left(u^{2}+v^{2}+1\right)^{2}, \quad(u, v) \in[0, \infty) \times[0, \infty) .
$$

Clearly, $f$ verifies (C3). Moreover, it is not difficult to see $f_{0}=\infty$, and accordingly the results of Theorem 3.2 are also valid.

At the end of the section, we list some related results and remarks.

Let us consider the multiparameter differential systems

$$
\left\{\begin{array}{l}
u^{\prime}(t)=-a_{1}(t) g_{1}(u(t)) u(t)+\lambda b_{1}(t) f\left(u\left(t-\tau_{1}(t)\right), v\left(t-\zeta_{1}(t)\right)\right), \\
v^{\prime}(t)=-a_{2}(t) g_{2}(v(t)) v(t)+\mu b_{2}(t) g\left(u\left(t-\tau_{2}(t)\right), v\left(t-\zeta_{2}(t)\right)\right),
\end{array}\right.
$$

where $\lambda, \mu>0$ are parameters. Under the same assumptions as before, one can check that system (3.3) is equivalent to

$$
\begin{aligned}
& u(t)=\lambda \int_{t}^{t+\omega} G_{1}(t, s) b_{1}(s) f\left(u\left(s-\tau_{1}(s)\right), v\left(s-\zeta_{1}(s)\right)\right) d s, \\
& v(t)=\mu \int_{t}^{t+\omega} G_{2}(t, s) b_{2}(s) g\left(u\left(s-\tau_{2}(s)\right), v\left(s-\zeta_{2}(s)\right)\right) d s,
\end{aligned}
$$

where

$$
G_{1}(t, s)=\frac{e^{\int_{t}^{s} a_{1}(\theta) g_{1}(u(\theta)) d \theta}}{e^{\int_{0}^{\omega} a_{1}(\theta) g_{1}(u(\theta)) d \theta}-1}, \quad G_{2}(t, s)=\frac{e^{\int_{t}^{s} a_{2}(\theta) g_{2}(v(\theta)) d \theta}}{e^{\int_{0}^{\omega} a_{2}(\theta) g_{2}(v(\theta)) d \theta}-1}, \quad t \leq s \leq t+\omega .
$$


Furthermore, by a similar argument as above, it is not difficult to see that the results of Theorems 3.1-3.3 remain true for system (3.3).

Remark 3.2 It is worth remarking that, under some reasonable assumptions, the results of the paper are still valid for the more general coupled systems

$$
u_{i}^{\prime}(t)+a_{i}(t) g_{i}\left(u_{i}(t)\right) u_{i}(t)=\lambda_{i} b_{i}(t) f_{i}\left(u_{1}\left(t-\tau_{i 1}(t)\right), \ldots, u_{n}\left(t-\tau_{\text {in }}(t)\right)\right), \quad i=1,2, \ldots, n
$$

and

$$
u_{i}^{\prime}(t)=a_{i}(t) g_{i}\left(u_{i}(t)\right) u_{i}(t)-\lambda_{i} b_{i}(t) f_{i}\left(u_{1}\left(t-\tau_{i 1}(t)\right), \ldots, u_{n}\left(t-\tau_{i n}(t)\right)\right), \quad i=1,2, \ldots, n .
$$

Acknowledgements

Not applicable.

\section{Funding}

The first author is supported by National Natural Science Foundation of China (Grant No. 61761002), First-Class Disciplines Foundation of Ningxia (No. NXYLXK2017B09), and the Key Project of North Minzu University (No. ZDZX201804).

\section{Availability of data and materials}

Not applicable.

\section{Competing interests}

The authors declare that they have no competing interests.

\section{Authors' contributions}

$\mathrm{RC}$ analyzed and proved the main results, and was a major contributor in writing the manuscript. XL checked the English grammar and typing errors in the full text. All authors read and approved the final manuscript.

\section{Publisher's Note}

Springer Nature remains neutral with regard to jurisdictional claims in published maps and institutional affiliations.

Received: 28 October 2019 Accepted: 21 January 2020 Published online: 30 January 2020

\section{References}

1. Chow, S.N.: Existence of periodic solutions of autonomous functional differential equations. J. Differ. Equ. 15, 350-378 (1974)

2. Wazewska-Czyzewska, M., Lasota, A.: Mathematical problems of the dynamics of a system of red blood cells. Mat. Stosow. 6, 23-40 (1976)

3. Gurney, W.S., Blythe, S.P., Nisbet, R.N.: Nicholson's blowflies revisited. Nature 287, 17-21 (1980)

4. Freedman, H.I., Wu, J.: Periodic solutions of single-species models with periodic delay. SIAM J. Math. Anal. 23, 689-701 (1992)

5. Kuang, Y.: Delay Differential Equations with Applications in Population Dynamics. Academic Press, New York (1993)

6. Mackey, M.C., Glass, L.: Oscillations and chaos in physiological control systems. Science 197, 287-289 (1997)

7. Jin, Z.L., Wang, H.Y.: A note on positive periodic solutions of delayed differential equations. Appl. Math. Lett. 23, 581-584 (2010)

8. Graef, J., Kong, L.J.: Existence of multiple periodic solutions for first order functional differential equations. Math. Comput. Model. 54, 2962-2968 (2011)

9. Ma, R.Y., Chen, R.P., Chen, T.L.: Existence of positive periodic solutions of nonlinear first-order delayed differential equations. J. Math. Anal. Appl. 384, 527-535 (2011)

10. Ma, R.Y., Lu, Y.Q.: One-signed periodic solutions of first-order functional differential equations with a parameter. Abstr. Appl. Anal. 2011, Article ID 843292 (2011)

11. Wang, H.Y.: Positive periodic solutions of functional differential systems. J. Differ. Equ. 202, 354-366 (2004)

12. Wang, H.Y.: Positive periodic solutions of singular systems of first order ordinary differential equations. Appl. Math. Comput. 218, 1605-1610 (2011)

13. Chen, R.P., Ma, R.Y., He, Z.Q.: Positive periodic solutions of first-order singular systems. Appl. Math. Comput. 218 , $11421-11428(2012)$

14. Zhang, G., Cheng, S.S.: Positive periodic solution of coupled delay differential systems depending on two parameters. Taiwan. J. Math. 4, 639-652 (2004)

15. Kiguradze, I., Puza, B.: On boundary value problems for systems of linear functional differential equations. Czechoslov. Math. J. 47, 341-373 (1997)

16. Kiguradze, I., Puza, B.: Boundary Value Problems for Systems of Linear Functional Differential Equations. Folia, Masaryk University, Brno (2003) 
17. Domoshnitsky, A., Hakl, R., Sremr, J.: Component-wise positivity of solutions to periodic boundary value problem for linear functional differential systems. J. Inequal. Appl. 2012, Article ID 112 (2012).

https://doi.org/10.1186/1029-242X-2012-112

18. Deimling, K.: Nonlinear Functional Analysis. Springer, Berlin (1985)

19. Guo, D.J., Lakshmikantham, V.: Nonlinear Problems in Abstract Cones. Academic Press, New York (1988)

Submit your manuscript to a SpringerOpen ${ }^{\circ}$ journal and benefit from:

- Convenient online submission

$\checkmark$ Rigorous peer review

- Open access: articles freely available online

- High visibility within the field

- Retaining the copyright to your article

Submit your next manuscript at $\boldsymbol{~ s p r i n g e r o p e n . c o m ~}$ 